\title{
81. On Deformations of Quintic Surfaces
}

\author{
By Eiji HoRIKAwA \\ University of Tokyo \\ (Comm. by Kunihiko KodaIrA, M. J. A., June 12, 1973)
}

Let $S_{0}$ be a non-singular hypersurface of degree 5 in the projective 3 -space $\boldsymbol{P}^{3}$ defined over $\boldsymbol{C}$. For brevity, we call $S_{0}$ a non-singular quintic surface.

By a surface, we shall mean a compact complex manifold of complex dimension 2, unless explicit indications are given. We say that a surface $S$ is a deformation of $S_{0}$ if there exists a finite sequence of surfaces $S_{0}, S_{1}, \cdots, S_{k}, \cdots, S_{n}=S$ such that, for each $k, S_{k}$ and $S_{k-1}$ belong to one and the same complex analytic family of surfaces.

If $S$ is a deformation of a non-singular quintic surface, $S$ has the following numerical characters:

$$
p_{g}=4, \quad q=0, \quad c_{1}^{2}=5,
$$

where $p_{g}, q$ and $c_{1}^{2}$ denote the geometric genus, the irregularity and the Chern number of $S$, respectively. In particular $S$ is an algebraic surface (see [5], Theorem 9). Moreover, since $S_{0}$ is minimal, Theorem 23 of Kodaira [5] asserts that

(**) $\quad S$ is minimal.

In this note, we shall give a statement of the results on the structures and deformations of surfaces which satisfy the conditions $(*)$ and $(* *)$. Details will be published elsewhere.

\section{Structures.}

Theorem 1. Let $S$ be a minimal algebraic surface with $p_{g}=4$, $q=0$, and $c_{1}^{2}=5$. Then the canonical system $|K|$ on $S$ has at most one base point. There are two cases:

Case I. $|K|$ has no base point. In this case, there exists a birational holomorphic map $f: S \rightarrow S^{\prime}$ of $S$ onto a (possibly singular) quintic surface $S^{\prime}$ in $\boldsymbol{P}^{3}$. $\quad S^{\prime}$ has at most rational double points as its singularities.

Case II. $|K|$ has one base point $b . \quad$ Let $\pi: \tilde{S} \rightarrow S$ be the quadric transformation with center at $b$. Then this case is divided as follows:

Case IIa. There exists a surjective holomorphic map $f: \tilde{S}$ $\rightarrow \boldsymbol{P}^{1} \times \boldsymbol{P}^{1}$ of degree 2 .

Case IIb. There exists a surjective holomorphic map $f: \tilde{S} \rightarrow \sum_{2}$ of degree 2, where $\sum_{2}$ denotes the Hirzebruch surface of degree 2, i.e., $\Sigma_{2}$ is a rational ruled surface with a section $\Delta_{0}$ with $\left(\Delta_{0}\right)^{2}=-2$.

The proof is based on a detailed analysis of the rational map $\Phi_{K}$ : 
$S \rightarrow \boldsymbol{P}^{3}$ defined by the canonical system $|K|$. The holomorphic maps $f$ in the above statement are derived from $\Phi_{K}$.

Corollary. If $|K|$ has a base point, then there exists a surjective holomorphic map $g: S \rightarrow \boldsymbol{P}^{1}$ whose general fibre is an irreducible nonsingular curve of genus 2 . In particular, the rational map $\Phi_{2 K}$ defined by the bicanonical system $|2 K|$ is not birational.

Conversely, we can construct every surface of type II as follows: First we construct a double covering $S^{\prime}$ of $W=\boldsymbol{P}^{1} \times \boldsymbol{P}^{1}$ or $\sum_{2}$ with appropriate branch locus on $W . S^{\prime}$ is a normal surface. Let $\tilde{S}$ be the minimal resolution of singularities of $S^{\prime}$ (see [1], p. 81). We construct $S^{\prime}$ so that $\tilde{S}$ contains one exceptional curve $E$. Contracting $E$ to a point, we obtain a minimal algebraic surface with $p_{g}=4, q=0$, and $c_{1}^{2}=5$.

2. Deformations. First, we give some results on small deformations of a surface in consideration.

Proposition 1. i) The classes of surfaces of type I and of type IIa are, respectively, closed under small deformations.

ii) A surface of type I and a surface of type IIa do not belong to one and the same family (with non-singular base space).

Theorem 2. Let $S$ be a surface of type IIb of which the canonical bundle is ample. Let $p: \mathcal{S} \rightarrow M$ be the Kuranishi family of deformations of $S=p^{-1}(0)$ with $0 \in M$ (see [7]). Then

i) $\quad M=M_{0} \cup M_{1}$ where each $M_{i}(i=0,1)$ is a 40-dimensional manifold,

ii) $\quad N=M_{0} \cap M_{1}$ is a 39-dimensional manifold,

iii) $S_{t}=p^{-1}(t)$ is a non-singular quintic surface, a surface of type IIa, or a surface of type IIb according as $t \in M_{0}-N, t \in M_{1}-N$, or $t \in N$.

We now indicate an outline of the proof of Theorem 2. Let $S$ denote a surface as in Theorem 2 and let $\Theta_{S}$ denote the sheaf of germs of holomorphic vector fields on $S$. We have $\operatorname{dim} H^{1}\left(S, \Theta_{S}\right)=41$ and $\operatorname{dim} H^{2}\left(S, \Theta_{S}\right)=1$. Let $D=\left\{t \in C^{41}:|t|<\varepsilon\right\}$ with $\varepsilon>0$ sufficiently small. Then there exists a $(0,1)$-form $\varphi(t)$ with coefficients in $\Theta_{S}$ depending holomorphically on $t \in D$ such that

$$
M=\{t \in D: \boldsymbol{H}[\varphi(t), \varphi(t)]=0\},
$$

where $\boldsymbol{H}$ denotes the projection onto the space of harmonic forms with respect to a Hermitian metric on $S$ and [ , ] denotes the Poisson bracket. Since $\operatorname{dim} H^{2}\left(S, \Theta_{S}\right)=1$, we may regard $H[\varphi(t), \varphi(t)]$ as a holomorphic function on $D$. We can prove that

$$
\boldsymbol{H}[\varphi(t), \varphi(t)]=t_{1} t_{2}+(\text { higher terms }),
$$

for an appropriate choice of coordinates $\left(t_{1}, t_{2}, \cdots, t_{41}\right)$ on $D$.

On the other hand, applying an improved form of Theorem $2^{\prime}$ of [3] to the holomorphic map $g: S \rightarrow \boldsymbol{P}^{1}$ in Corollary to Theorem 1 , we can 
construct a 40-dimensional effectively parametrized family $p_{1}: \mathcal{S}_{1} \rightarrow M_{1}$ of deformations of $S=p_{1}^{-1}(0)$ with $0 \in M$ (see [6], Definition 6.4).

It follows that $\boldsymbol{H}[\varphi(t), \varphi(t)]$ decomposes into a product $q(t) r(t)$ with $q(t)=t_{2}+\left(\right.$ higher terms), $r(t)=t_{1}+$ (higher terms). This proves the assertion i).

Other assertions can be proved by applying the general theory on deformations of holomorphic maps [4].

It seems difficult to study the deformations of a surface of which the canonical bundle is not ample. However, applying a result of Brieskorn ([1], [2]), we can prove

Theorem 3. Every minimal algebraic surface with $p_{g}=4, q=0$, and $c_{1}^{2}=5$, is a deformation of a non-singular quintic surface.

\section{References}

[1] Brieskorn, E.: Über die Auflösung gewisser Singularitäten von holomorphen Abbildungen. Math. Ann., 166, 76-102 (1966).

[2] —-: Die Auflösung der rationalen Singularitäten holomorpher Abbildungen. Math. Ann., 178, 255-270 (1968).

[ 3 ] Horikawa, E.: On deformations of holomorphic maps. Proc. Japan Acad., 48, 52-55 (1972).

[4] - On deformations of holomorphic maps. I (to appear in J. Math. Soc. Japan, 25 (1973)), II (in preparation).

[5] Kodaira, K.: On the structure of compact complex analytic surfaces. I. Amer. J. Math., 86, 751-798 (1964).

[6] Kodaira, K., and Spencer, D. C.: On deformations of complex analytic structures. I, II. Ann. of Math., 67, 328-466 (1958).

[ 7 ] Kuranishi, M.: On the locally complete families of complex analytic structures. Ann. of Math., 75, 536-577 (1962). 